\title{
The role of dermoscopy in dermato-oncological diagnostics - new trends and perspectives
}

\author{
Grażyna Kamińska-Winciorek, Aleksandra Pilśniak²
}

\author{
'Skin Cancer and Melanoma Team, Department of Bone Marrow Transplantation and Oncohematology, M. Sklodowska-Curie National Research Insti- \\ tute of Oncology, Gliwice Branch, Poland \\ 2Inpatient Department of Radiation and Clinical Oncology, M. Sklodowska-Curie National Research Institute of Oncology, Gliwice Branch, Poland
}

Medical history and clinical examination are the most basic elements of medical diagnostics. Clinical examination in the context of dermatology should be combined with the taking and archiving of clinical, dermoscopic and/or video dermoscopic photographs. Dermoscopy is a non-invasive examination and is the recommended method of examining skin lesions. It requires many years of experience and extensive training, and subsequently can be very helpful in the diagnostic process since it allows for a more thorough examination than the unarmed eye. The diagnosis of malignant skin tumours has been significantly improved by noninvasive real-time diagnostic devices. Based on the data from the literature available, we discussed the most commonly used algorithms in the diagnostic process. It should be emphasized that a dermoscopic evaluation may facilitate the diagnosis and early treatment of micromelanoma and basal cell carcinoma. Finally, the role of dermoscopy in the follow-up procedure of oncologic patients should not be forgotten.

Key words: dermoscopy, dermato-oncology, skin cancer, cutaneous melanoma, skin malignancies

\section{Introduction}

Medical history and clinical examination are the most basic elements of medical diagnostics. It should be emphasized that a clinical examination in the context of dermatology should be combined with taking and archiving of clinical, dermoscopic and/or videodermoscopic photographs [1, 2]. Dermoscopy is a non-invasive examination and it is the recommended method of examining skin lesions since it allows for a more thorough examination than the unarmed eye. This diagnostic tool has several uses. The first one is self training, when a specific diagnosis is straightforward. In this case, this method provides us with an enormous amount of data. We are able to correlate our macroscopic thinking with the dermoscopic image, which consequently broadens our knowledge. In the second situation, a diagnosis is very likely and we use a dermoscope to confirm our assumptions and this ensures we can refrain from performing a biopsy. In the next case, a dermoscopy reverses the diagnosis and corrects mistakes. In the latter case, a dermoscopy can lead to a diagnosis by visualizing the feature, resulting in a list of differential diagnoses.

\section{Diagnosis of malignant skin tumours}

The diagnosis of malignant skin tumours has been significantly improved by noninvasive real-time diagnostic devices. It is obvious that such a diagnosis must be confirmed by histopathological diagnosis [3]. Dermoscopy requires several years of experience and extensive training, and subsequently can be very helpful in the diagnostic process leading to the final confirmation in the form of a histopathological examination [4]. Consequently, it is worth mentioning and characterizing the

\section{How to cite:}

Kamińska-Winciorek G, Pilśniak A. The role of dermoscopy in dermato-oncological diagnostics-new trends and perspectives. NOWOTWORY J Oncol 2021; 71: 103-1 10. 
classic patterns of the most common skin cancer, i.e. basal cell carcinoma (BCC) in dermoscopy. Undoubtedly, the presence of arborizing vessels, large blue-grey ovoid nests, ulceration, leaf-like areas and spoke wheel-like structures and numerous blue-grey globules indicate the basal cell carcinoma (fig. 1A, B) [5].

The second diagnosis we should look at is melanoma. We observe an increasing number of algorithms that help in the early diagnosis of melanoma which are listed and described below. We have dealt with the differences between patients with a solitary lesion, of which a surgical excision is the best procedure. On the other hand, there are patients with numerous lesions which cannot all be cut out; in this case, a dermoscopy with computerized photo archiving is very useful. In addition to tumour diagnosis, the morphological features of the tumour may be important in designing a treatment strategy. It is suggested that the presence of multiple minor erosions or ulceration is a crucial predictor of basal cell carcinomas'

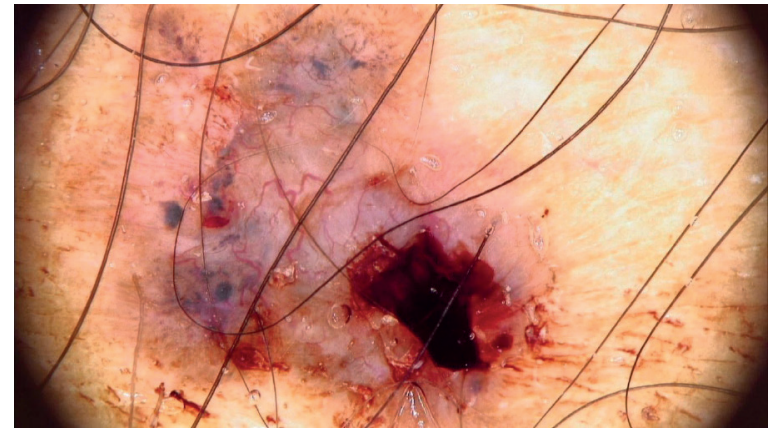

Figure 1A. Dermoscopic features in a non-polarized dermoscopy (NPD) of basal cell carcinoma include the presence of arborizing vessels (bright red, thick diameter vessels $(0.2 \mathrm{~mm}$ or more) from which emanate branching vessels with progressively thinner diameters), large blue-grey ovoid nests (confluent, well-circumscribed, pigmented ovoid areas), multiple blue-grey dots (pinpoint blue-grey structures) and globules (well-defined round or oval structures), ulceration (shallow erosions that may be covered with congealed blood). Dermoscopic definitions based on dermoscopedia.org [49]

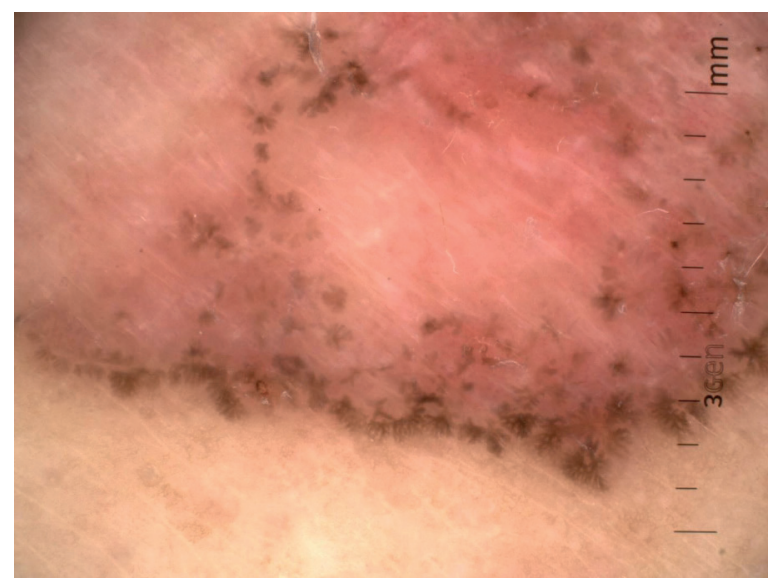

Figure 1B. Dermoscopy in a polarized dermoscopy (PD) of basal cell carcinoma indicates the presence of leaf-like structures (linear to bulbous extensions connected at an off-center base area) and spoke wheel-like structures (radial projections that surround a central darker point). Moreover, in the centre of the lesion shiny white strands (parallel and linear white areas that do not usually intersect) are noticed. Dermoscopic definitions based on dermoscopedia.org [49] response to imiquimod and the presence of pigmentation is a negative predictor of a worse response of this cancer to photodynamic therapy $[6,7]$.

\section{Algorithms for melanocytic lesions}

A dermoscopic examination performed by experienced doctors is more accurate than the clinical examination itself. In the study of the observed features visible in a dermoscopy, many algorithms have been established that allow an approximation of an accurate diagnosis. The most commonly used algorithms are discussed below. Kamińska-Winciorek et al. in their review present in detail the older algorithms widely previously used and described in literature [8].

\section{Three-Point Checklist}

The Three-Point Checklist algorithm takes into account three criteria to which it belongs:

1. asymmetry in dermoscopic structures' distribution,

2. an atypical pigmented network and

3. blue-white structures.

This Three-Point Checklist can be used by clinicians in diagnostics not only for melanoma (fig. 2A) but also basal cell carcinoma [9]. Soyer et al. showed that the presence of either of these two criteria indicates a high probability of melanoma [9].

\section{Seven-Point Checklist}

The Seven-Point Checklist algorithm includes seven characteristics, including: atypical pigment network, gray-blue areas, atypical vascular pattern, radial streaming (streaks), irregular diffuse pigmentation (blotches), irregular dots and globules, regression pattern (a presence of white scar-like depigmentation or peppering known as multiple scattered blue-grey granules) (fig. 2B). Historically, a minimum score of three for adding individual features of the above-mentioned seven is required for the diagnosis of melanoma [10]. Previously, at least

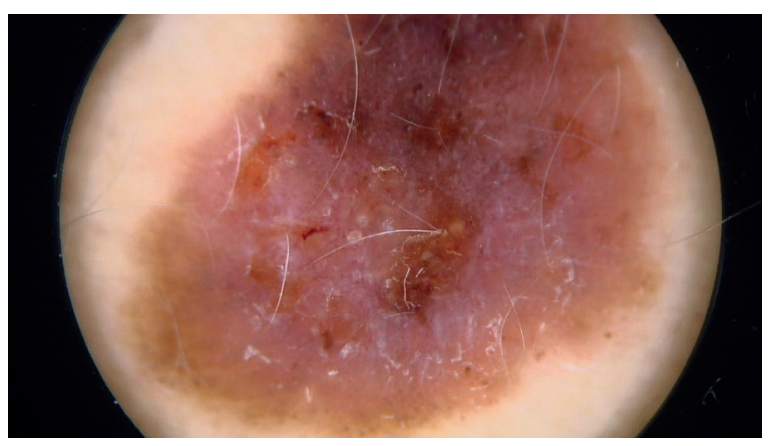

Figure 2A. Dermoscopic assessment of a superficial spreading melanoma (SSM) according to the Three-Point Checklist reveals the presence of asymmetry in dermoscopic structures' distribution (according to two axes), an atypical pigmented network and blue-white structures. Moreover, white structures which are seen in the presented case of SSM in polarized light, so-called shiny white streaks (former synonyms: chrysalis - chrysalids - crystalline) in definition as lines, white, perpendicular shiny white streaks usually correspond with invasive type of melanomas. Dermoscopic definitions based on dermoscopedia.org [49] 


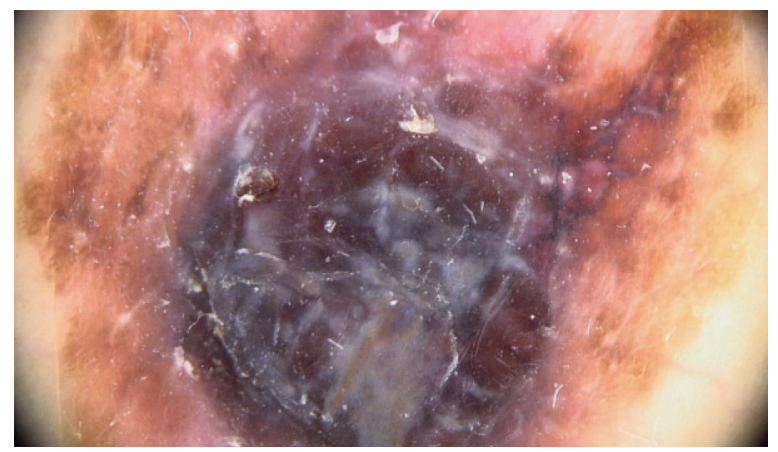

Figure 2B. Dermoscopy of a nodular melanoma in polarized light. The Seven-Point Checklist algorithm indicates the presence of 7 characteristic features, including: atypical pigment network, grey-blue areas, atypical vascular pattern, radial streaming (streaks), irregular diffuse pigmentation (blotches), irregular dots and globules, regression pattern. Moreover, multiple shiny white streaks and strands corresponding with deep dermal fibrosis are visible

two dermoscopic criteria (one major and one minor) must be present for a suspicious diagnosis (a score of three or more). In 2011, Argenziano et al. revised Seven-Point Checklist. They showed in their study that in order to increase the sensitivity of the assessment in the Seven-Point Checklist, the excision threshold of the lesion should be adjusted compared to the original [11]. In the revised Seven-Point Checklist, each criterion receives 1 point, the notch threshold is 1 point, not 3 points like in the earlier version [11].

\section{Two Step Algorithm}

In the previous traditional two-step algorithm, assessment is divided into two steps including the differentiation between melanocytic and non-melanocytic changes. When the lesion is classified as melanocytic, the observer then proceeds to the second stage consisting in qualifying the change as mild or malignant. During this second step a decision must be made whether the melanocytic lesion is benign, suspect, or malignant. For this purpose, the mentioned algorithms can be useful, including pattern analysis, ABCD rule, Menzies method and the Seven-Point Checklist which was discussed above $[12,13]$.

Pattern analysis is a method that involves assessing all the dermoscopic features that a lesion shows. In general terms, malignant - suspected lesions have several colors that are disordered in structure and are asymmetrical in dermoscopic distribution. The $A B C D$ rule of dermoscopy is based on the following criteria: asymmetry (A), border (B), colour (C) and differential structures (D) [14].

The Menzies method aims to distinguish between benign lesions and melanomas. This method includes negative features (symmetrical pattern, single color) indicating benign changes and positive features indicating melanoma. The positive features include blue-white veil, multiple brown dots, pseudopods, radial streaming, scar-like depigmentation, multiple (5-6) colors, multiple blue/grey dots, broadened ne- twork [15]. Exceptions to the two-step algorithms have been observed over the years. Moreover hybrid dermoscopes allow the user to toggle between polarized and non-polarized light and consequently a diagnosis becomes more likely. Some dermoscopic structures are more prominent in non-polarized dermoscopy (NPD) and others in polarized (PD) [16]. In 2010, an update of this 2-step algorithm was proposed, which consists in adding 2 decision levels to help doctors correctly classify some of the so-called featureless neoplasms as melanocytic or non-melanocytic tumours. In the revised two-step algorithms, the main queries of conducted analysis is to establish a specific diagnosis (step 1) and to rule out melanoma (step 2). This algorithm impedes the use of unpolarized dermoscopy [17].

\section{Triage Amalgamated Dermoscopic Algorithm (TADA)}

It is worth noting that the algorithms mentioned so far have been used to detect specific subsets of pigmented skin neoplasms - mainly pigmented melanoma. This is a limitation of these algorithms because many melanomas, basal cell carcinomas and squamous cell carcinomas do not have this pigment. Thus, compared to the above algorithms, the TADA algorithm allows the identification of pigmented and non-pigmented skin malignancies. At the very beginning, this algorithm requires the exclusion of three common and clearly benign lesions, i.e. cherry haemangioma (fig. 3A), dermatofibroma (fig. 3B) or seborrheic keratosis (fig. 3C. In the next step, dermoscopic patterns are taken into account, i.e. the distribution of colours and structures within the lesion. If there is an architectural disorder/disorganized pattern, a biopsy should be performed. If we have organized lesions with a starburst pattern (fig. 3D) or with any of the following features: blue-black/grey colour, shiny white structures, negative network, ulcer/erosion, vessels (fig. 3E, F) a biopsy should be performed [18, 19].

\section{Metaphoric and descriptive terminology}

According to Blum et al., the more metaphorical assessment called blink and more descriptive one colloquially called think complement each other and are used all over the world [20]. However, in a clinical and scientific context, clear and universal language should be the basis. In 2016, Kittler et al. published a consensus aimed at standardizing the dermoscopic description [21].

\section{Early detection of micro-melanoma and basal cell carcinoma}

We should pay attention to the change of the type of micro-melanoma, which, due to its size, i.e. $5 \mathrm{~mm}$, does not meet the criterion D of the ABCD assessment and is often overlooked. In this case, a dermoscopic evaluation may facilitate diagnosis and early treatment. So far, there are very few published studies evaluating micro-melanomas. Megaris et al. in their retrospective study suggest features that increase the probability 
A

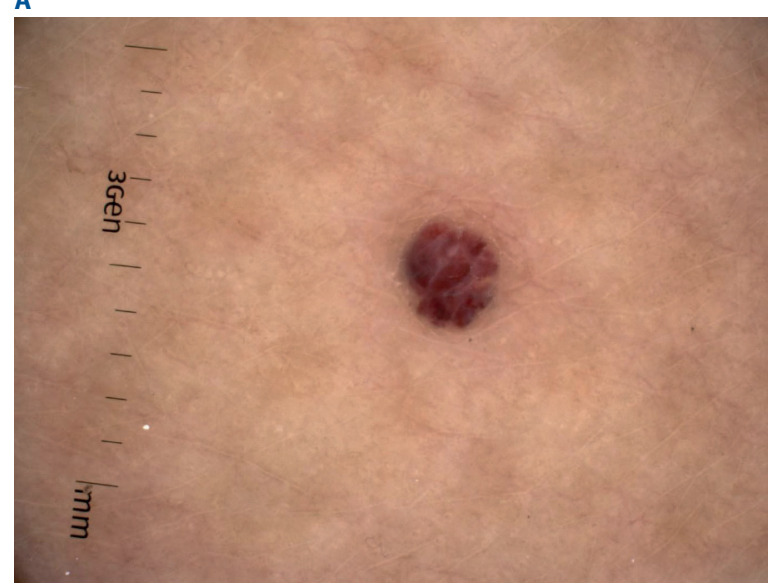

C

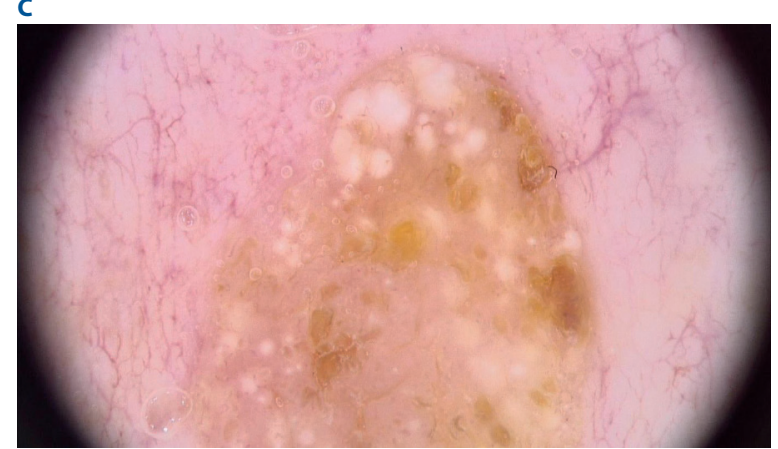

E

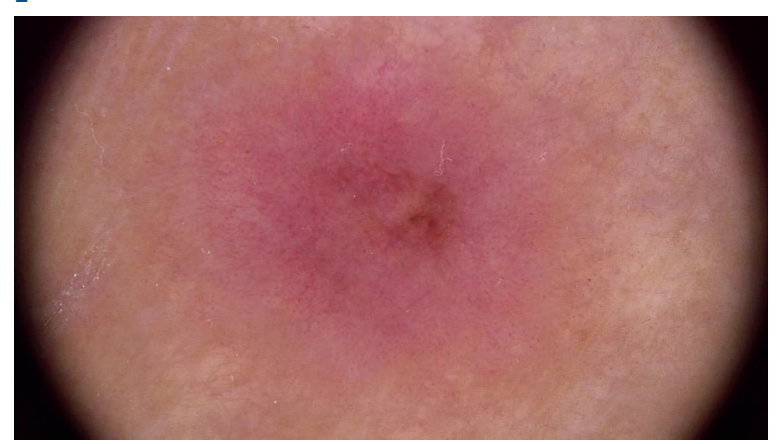

B

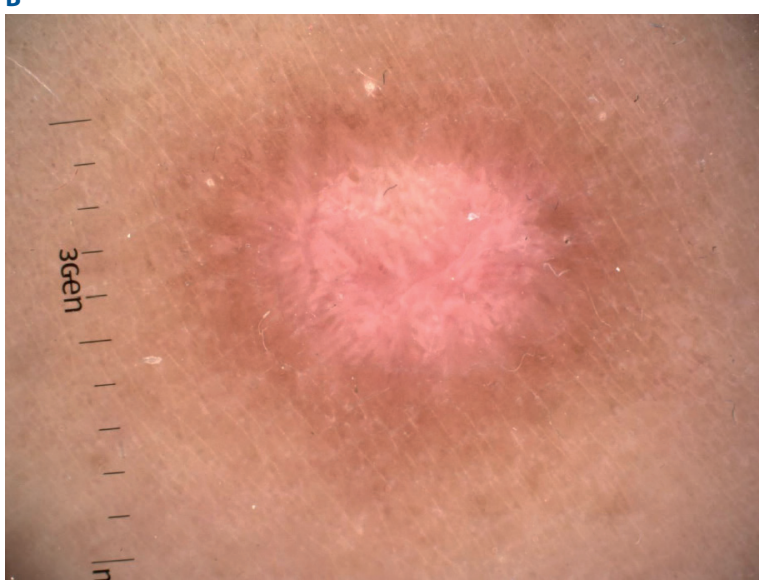

D
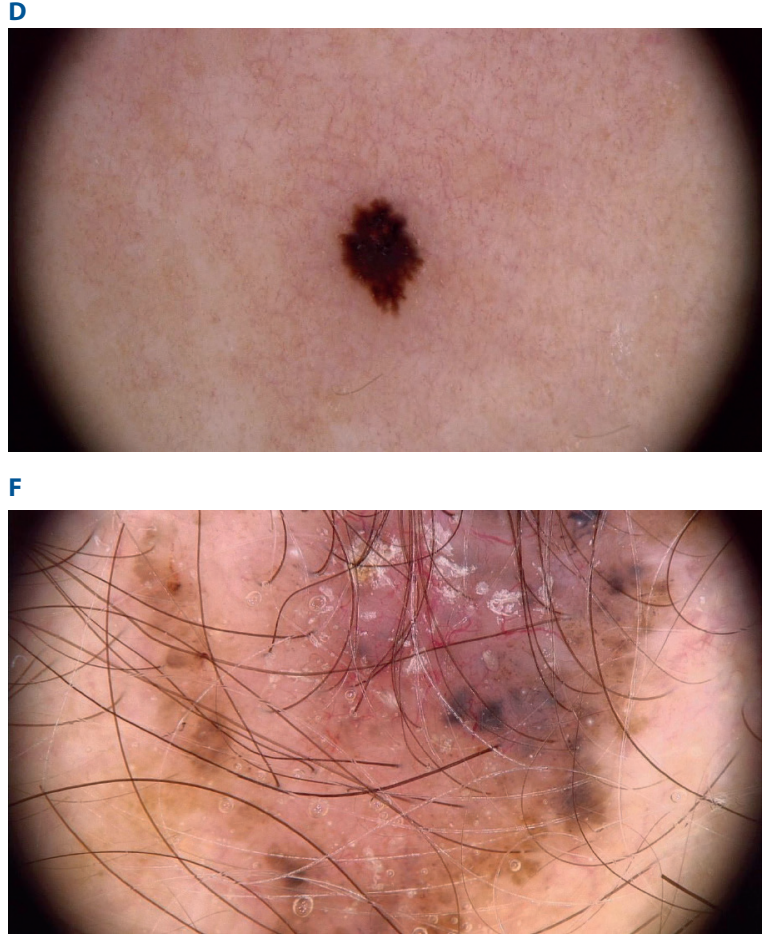

Figure 3. At the very beginning, theTriage Amalgamated Dermoscopic Algorithm (TADA) requires the exclusion of three common and clearly benign lesions; A - cherry haemangioma (with the presence of lacunae defined as round to oval red, reddish-brown or reddish-blue areas that commonly vary in size and colour - PD); B - dermatofibroma (the peripheral network with a central white scar-like area with a pink hue and shiny white lines in polarized light) or C - seborrheic keratosis (with multiple dots or clods white disseminated in NPD). In the TADA algorithm, if we have organized lesions with D - a starburst pattern (typified by streaks, pseudopods, or finger-like projections regularly distributed on the periphery; Reed nevus in NPD) or any of the following features: $\mathbf{E}$ - vessels (multiple dotted and linear irregular vessels in SSM in NPD); $\mathbf{F}$ - blue-black/grey colour (BCC in NPD), negative network, shiny white structures, ulcer/erosion, a biopsy should be performed

of malignancy in lesions up to $5 \mathrm{~mm}$. Such features include irregular hyperpigmented areas, atypical dots/globules, and an atypical network, within a reticular or unstructured global pattern (fig. 4A) [22].

The routine use of dermoscopy allows the detection of melanomas of which patients are unaware [23]. Moreover, the digital follow-up enables recognition of early melanoma when specific structures or criteria for malignancy may not be present [24]. The combined use of total-body photography and sequential digital dermoscopy enables the detection of incipient melanomas that might have been overlooked if assessed solely by the naked eye [23, 24]. Moreover most melanomas are diagnosed with digital dermoscopy monitoring by side-by-side image comparison [25].

Dermoscopy can also aid early diagnosis of small basal cell carcinomas less than $5 \mathrm{~mm}$ in diameter, especially characterized newly arised lesions located on the skin of the head and neck [26]. They are characterized by the presence of multiple blue grey dots and large blue-grey ovoid nests [26] especially in its pigmented variants of very small BCC (3 mm-sized) (fig. 4B) [27]. Moreover the presence of arborizing vessels with the existence of shiny white blotches and strands may also help 
can the BCC recognition although 1/3 of small lesions did not exhibit the typical dermoscopic criteria of BCC [28]. It is evident that in small size BCC classic dermoscopic criteria (the presence of arborizing vessels and ulceration) are often substituted by non-classical criteria [29]. Only blue-whitish veil and blue in-focus dots dermoscopic features among non-classic criteria

\section{A}

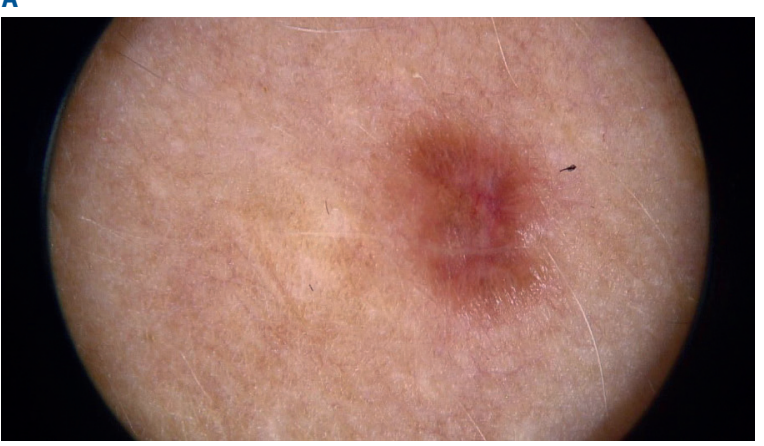

B
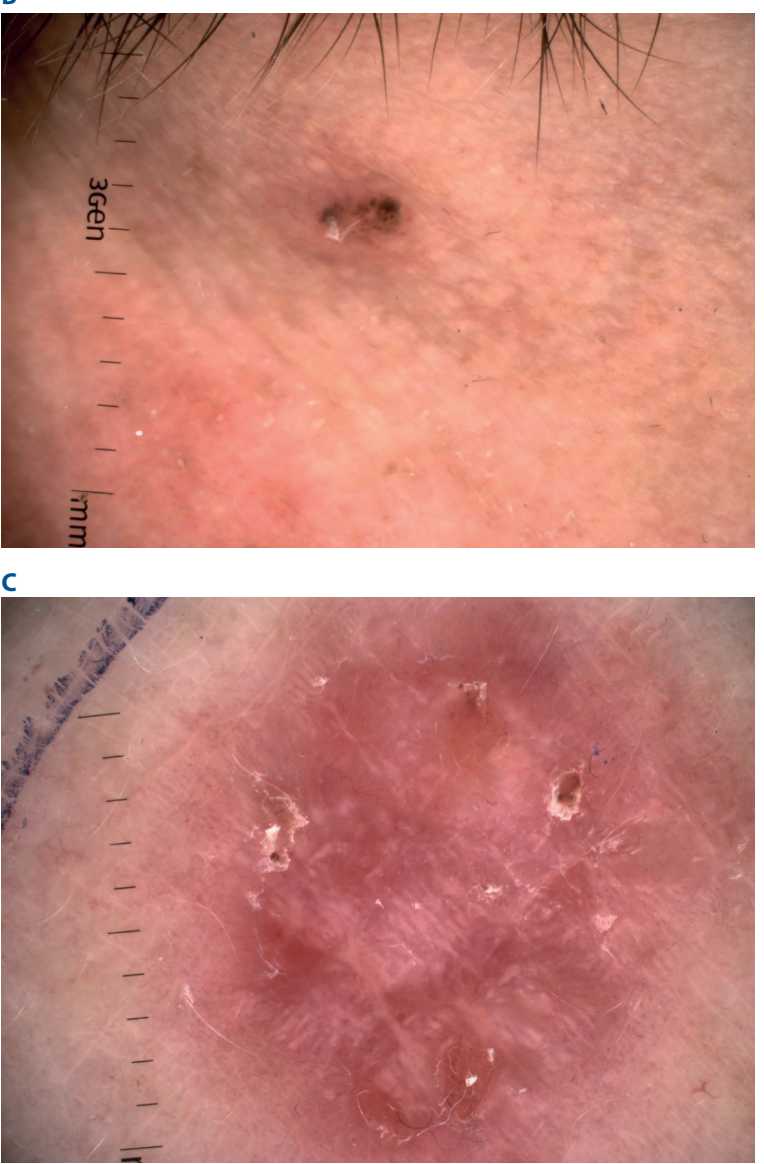

Figure 4. A - a micro-melanoma measuring $3 \mathrm{~mm}$ proved histopathologically as SSM located on the décolletage. Dermoscopy in polarized light exhibits the presence of short shiny white streaks and an atypical network, within an unstructured global pattern; B - small basal cell carcinoma sized less than $2 \mathrm{~mm}$ in diameter located on the skin of the face, characterized by the presence of multiple blue grey dots and globules; $\mathbf{C}$ - non-classic BCC criteria include inter alia: pink-white areas with: white strands (bright-white less well defined lines, oriented parallel or distributed haphazardly) and shiny white blotches (as white structures in the form of large areas, clods or circles), micro-erosions (covered by crusts and blood) and short fine telangiectasias seen in polarized dermoscopy. Dermoscopic definitions based on dermoscopedia.org [49] which represent the neoplasm's early phase indicated a good agreement among low experience observers [29].

\section{Dermoscopic follow-up in dermato-oncology}

\section{Dermoscopic assessment of the surgical margins before excision}

Preoperative digital dermoscopy is a better method for detecting tumoral margins than clinical evaluation, and is an effective, simple, non-invasive method for the pre-surgical evaluation of margins [30]. Preoperative dermoscopy is a better method to determine the margins of neoplasms than clinical evaluation alone [31]. Moreover, the preoperative dermoscopic assessment using non-classic BCC criteria including pink-white areas and short telangiectasias in the area between clinically and dermoscopically detected margins, helps define the neoplasm's margins and to achieve a really radical excision (fig. 4C) [32].

\section{Dermoscopic follow-up after surgical procedures}

Dermoscopy, as a non-invasive method, works well in secondary prevention, i.e., early detection of neoplasms with the use of dermoscopic assessment of the entire skin, covering areas that are difficult to access during the examination. We should emphasize the importance of this method in the follow-up stages of patients after cancer treatment. These are high-risk patients at risk of relapse and should be regularly monitored using the above method along with image archiving. Dermoscopic follow-up is used in the control of post-excision malignant tumour scars enabling the diagnosis and assessment of tumour (eg. lentigo maligna melanoma - LMM) persistence after surgery (fig. 5A) [33], rapid recognition of the features of tumour recurrence among others, melanoma within the scar (fig. 5B) [34] with an assessment of its healing or leaving sutures (fig. 5C). In addition, a dermoscopic observation of the whole body of patients with diagnosed malignant neoplasms enables early detection of metastases the nature of satellitosis, in-transit (fig. 5D) or distant localized within the skin and subcutaneous tissue $[35,36]$ as well as allowing for additional monitoring dermoscopic effects of the therapies used in patients with, inter alia, metastatic melanoma (blood vessel morphology and distribution, degree of vascularization, ulceration, background). Dermoscopy is also used in patients diagnosed with cutaneous malignancies for the early detection of synchronous melanoma [37,38] and basal or squamous cell carcinoma (SCC) with dermoscopic assessment of the selected therapies of skin cancers.

\section{Dermoscopic assessment of the selected therapies of skin cancers}

Moreover, patients' response to treatment can be easily monitored with this noninvasive medical device, thus allowing further modulation of the therapy [4]. It is worth mentioning 
A

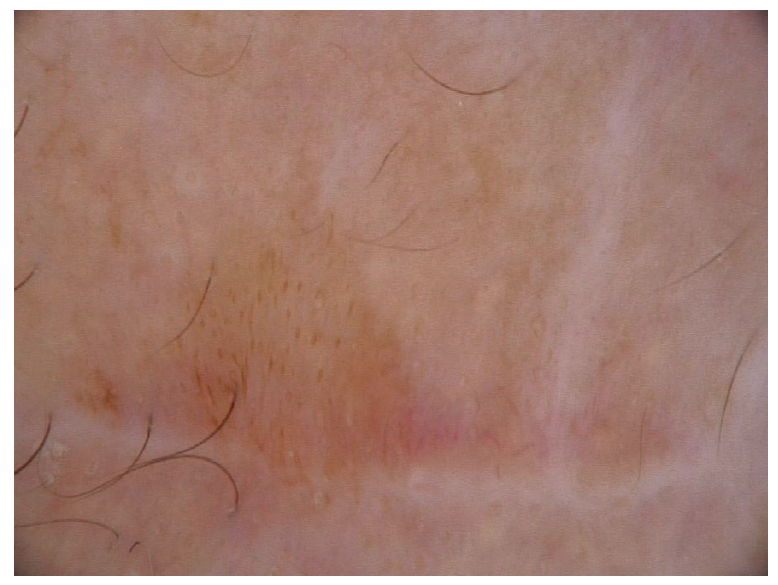

C

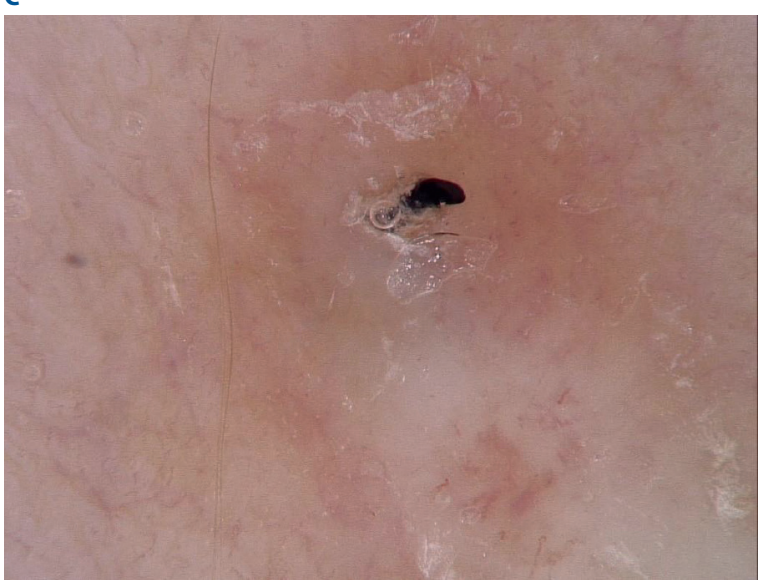

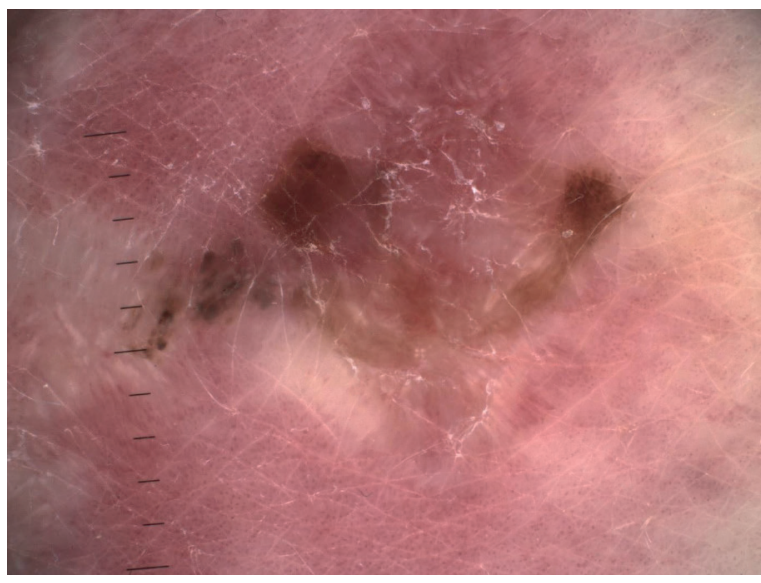

D

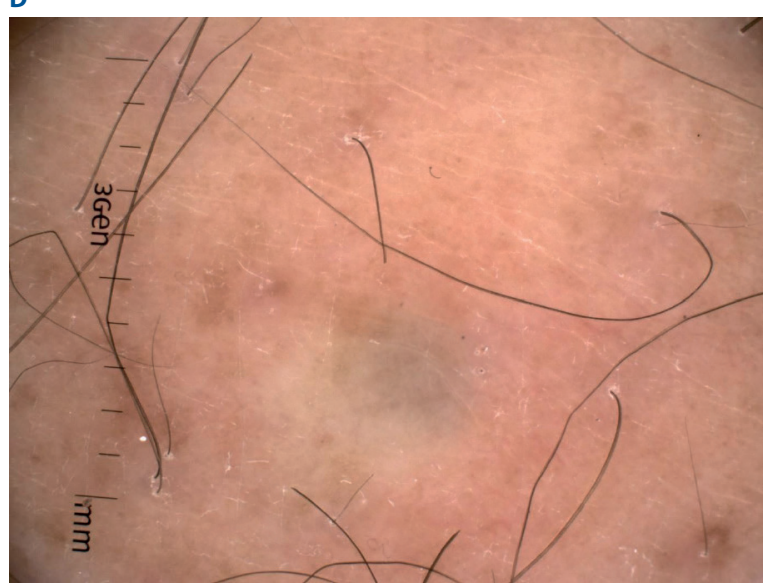

Figure 5. Dermoscopic follow-up in the control of post-excision scars of malignant tumours enabling diagnosis and the assessment of tumour persistence after surgery; $\mathbf{A}$ - lentigo maligna in NPD (with a pattern of hyperpigmented follicular openings as fine circles and semicircles), rapid recognition of the features of tumour recurrence; $\mathbf{B}$ - thick melanoma within the scar (the presence of an atypical pigmented network and irregular grey and brown clods, PD); C - assessment of leaving sutures (black-blue solitary clod corresponding with a non-absorbable suture within the scar, NPD); D - according to melanoma metastasis, dermoscopic classification [35] distinguish four dermoscopic patterns based on metastases' colour: blue, pink, brown and mixed pattern. The blue pattern of in-transit melanoma metastasis revealed the presence of structureless bluish areas in polarized dermoscopy

the treatment with the use of appropriate methods that can be considered and applied in the case of BCC and SCC, characterized by low risk of recurrence or in patients with contraindications to the use of basic methods such as surgery. Imiquimod (5\%) is used in the treatment of actinic keratosis, in situ SCC/Bowen's disease, and non-invasive forms of superficial spreading BCC [39]. Based on the Husein-EIAhmed study, dermoscopic evaluation improves the accuracy of the assessment of clinical response to imiquimod in pigmented BCC [40].

Dermoscopic follow-up was useful in monitoring the therapeutic response to selected topical therapies including ingenol mebutate in BCC [41], Bowen's disease [42] and imiquimod in LMM [33] as well as systemic therapy with vismodegib in BCC [43]. Dermoscopy was also used in monitoring BCC's treatment effects using high dose ionizing radiation therapy [44], changes in the course of LMM radiotherapy [45], or dermoscopic margin delineation in radiotherapy planning for superficial or nodular basal cell carcinoma [46]. In addition, the dermoscope can be used to assess skin toxicity or lesions occurring in existing and newly formed melanocytic changes during the treatment of melanoma, including with the use of BRAF inhibitors $[47,48]$.

\section{Conclusions}

Modern perspectives regarding dermoscopy emphasize its multidisciplinary scope and nature concerning not only the preoperative diagnosis of skin cancers but also the post-operative and post-therapeutic stages - including topical and systemic implemented therapies.

The high-resolution illustrations are available in the electronic version of this article in the Supplemetary materials section on the website nowotwory.edu.pl.

Conflict of interest: none declared 


\section{Grażyna Kamińska-Winciorek}

M. Sklodowska-Curie National Research Institute of Oncology Gliwice Branch

Department of Bone Marrow Transplantation

and Oncohematology

ul. Wybrzeże Armii Krajowej 15

44-101 Gliwice, Poland

e-mail: grazyna.kaminskawinciorek@gmail.com

\section{Received: 4 Jan 2021}

Accepted: 11 Jan 2021

\section{References}

1. Kamińska-Winciorek G, Gajda M, Wydmański J, et al. What do Web users know about skin self-examination and melanoma symptoms? Asian Pac J Cancer Prev. 2015; 16(7): 3051-3056, doi: 10.7314/ apjcp.2015.16.7.3051, indexed in Pubmed: 25854404.

2. Forsea AM, TschandI P, Zalaudek I, et al. Eurodermoscopy Working Group. The impact of dermoscopy on melanoma detection in the practice of dermatologists in Europe: results of a pan-European survey. J Eur Acad Dermatol Venereol. 2017; 31(7): 1148-1156, doi: 10.1111/ jdv.14129, indexed in Pubmed: 28109068.

3. Rutkowski P, Wysocki PJ, Nasierowska-Guttmejer A, et al. Cutaneous melanoma. Oncol Clin Pract. 2020; 16(4): 163-182, doi: 10.5603/ OCP.2020.0021.

4. Bakos RM, Blumetti TP, Roldán-Marín R, et al. Noninvasive Imaging Tools in the Diagnosis and Treatment of Skin Cancers. Am J Clin Dermatol. 2018; 19(Suppl 1): 3-14, doi: 10.1007/s40257-018-0367-4, indexed in Pubmed: 30374899

5. Que SK. Research Techniques Made Simple: Noninvasive Imaging Technologies for the Delineation of Basal Cell Carcinomas. J Invest Dermatol. 2016; 136(4): e33-e38, doi: 10.1016/j.jid.2016.02.012, indexed in Pubmed: 27012561.

6. Lallas A, Apalla Z, Argenziano G, et al. The dermatoscopic universe of basal cell carcinoma. Dermatol Pract Concept. 2014; 4(3): 11-24, doi: 10.5826/dpc.0403a02, indexed in Pubmed: 25126452.

7. Russo T, Piccolo V, Lallas A, et al. Dermoscopy of Malignant Skin Tumours: What's New? Dermatology. 2017; 233(1): 64-73, doi: 10.1159/000472253, indexed in Pubmed: 28486238.

8. Kamińska-Winciorek G, Spiewak R. [Basic dermoscopy of melanocytic lesions for beginners]. Postepy Hig Med Dosw (Online). 2011; 65: 501508, doi: 10.5604/17322693.955121, indexed in Pubmed: 21918252.

9. Soyer HP, Argenziano G, Zalaudek I, et al. Three-point checklist of dermoscopy. A new screening method for early detection of melanoma. Dermatology. 2004; 208(1): 27-31, doi: 10.1159/000075042, indexed in Pubmed: 14730233.

10. Argenziano G, Fabbrocini G, Carli P, et al. Epiluminescence microscopy for the diagnosis of doubtful melanocytic skin lesions. Comparison of the $A B C D$ rule of dermatoscopy and a new 7-point checklist based on pattern analysis. Arch Dermatol. 1998; 134(12): 1563-1570, doi: 10.1001/archderm.134.12.1563, indexed in Pubmed: 9875194.

11. Argenziano $G$, Catricalà $C$, Ardigo $M$, et al. Seven-point checklist of dermoscopy revisited. Br J Dermatol. 2011; 164(4): 785-790, doi: 10.1111/j.1365-2133.2010.10194.x, indexed in Pubmed: 21175563.

12. Scope A, Benvenuto-Andrade C, Agero AL, et al. Nonmelanocytic lesions defying the two-step dermoscopy algorithm. Dermatol Surg. 2006; 32(11): 1398-1406, doi: 10.1111/j.1524-4725.2006.32312.x, indexed in Pubmed: 17083595.

13. Argenziano G, Soyer HP, Chimenti S, et al. Dermoscopy of pigmented skin lesions: results of a consensus meeting via the Internet. J Am Acad Dermatol. 2003; 48(5): 679-693, doi: 10.1067/mjd.2003.281, indexed in Pubmed: 12734496.

14. Stolz W, Riemann $A$, Cognetta $A B$, et al. $A B C D$ rule of dermatoscopy: a new practical method for early recognition of malignant melanoma. Eur J Dermatol. 1994: 4521-4527.

15. Menzies SW, Ingvar C, Crotty KA, et al. Frequency and morphologic characteristics of invasive melanomas lacking specific surface microscopic features. Arch Dermatol. 1996; 132(10): 1178-1182, indexed in Pubmed: 8859028.

16. Braun RP, Scope A, Marghoob AA. The „blink sign” in dermoscopy. Arch Dermatol. 2011; 147(4): 520, doi: 10.1001/archdermatol.2011.82, indexed in Pubmed: 21482914.
17. Marghoob AA, Braun R. Proposal for a revised 2-step algorithm for the classification of lesions of the skin using dermoscopy. Arch Dermatol. 2010; 146(4): 426-428, doi: 10.1001/archdermatol.2010.41, indexed in Pubmed: 20404234.

18. Seiverling EV, Ahrns HT, Greene A, et al. Teaching Benign Skin Lesions as a Strategy to Improve the Triage Amalgamated Dermoscopic Algorithm (TADA). J Am Board Fam Med. 2019; 32(1): 96-102, doi: 10.3122/ jabfm.2019.01.180049, indexed in Pubmed: 30610147.

19. Jaimes N, Marghoob AA. Triage amalgamated dermoscopic algorithm. J Am Acad Dermatol. 2020; 82(6): 1551-1552, doi: 10.1016/j. jaad.2020.01.079, indexed in Pubmed: 32045619.

20. Blum A, Argenziano G. Metaphoric and descriptive terminology in dermoscopy: Combine „blink" with „think". Dermatol Pract Concept. 2015; 5(3): 23, doi: 10.5826/dpc.0503a05, indexed in Pubmed: 26336619.

21. Kittler H, Marghoob AA, Argenziano G, et al. Standardization of terminology in dermoscopy/dermatoscopy: Results of the third consensus conference of the International Society of Dermoscopy. J Am Acad Dermatol. 2016; 74(6): 1093-1106, doi: 10.1016/j.jaad.2015.12.038, indexed in Pubmed: 26896294.

22. Megaris A, Lallas A, Bagolini LP, et al. Dermoscopy features of melanomas with a diameter up to $5 \mathrm{~mm}$ (micromelanomas): A retrospective study. J Am Acad Dermatol. 2020; 83(4): 1160-1161, doi: 10.1016/j. jaad.2020.04.006, indexed in Pubmed: 32289392.

23. Salerni G, Alonso C, Fernández-Bussy R. Multiple Primary Invasive Small-Diameter Melanomas: Importance of Dermoscopy and Digital Follow-up. Dermatol Pract Concept. 2019; 9(1): 69-70, doi: 10.5826/ dpc.0901a16, indexed in Pubmed: 30775153.

24. Salerni G, Alonso C, Fernández-Bussy R. A series of small-diameter melanomas on the legs: dermoscopic clues for early recognition. Dermatol Pract Concept. 2015; 5(4): 31-36, doi: 10.5826/dpc.0504a08, indexed in Pubmed: 26693087.

25. Babino G, Lallas A, Agozzino M, et al. Melanoma diagnosed on digital dermoscopy monitoring: A side-by-side image comparison is needed to improve early detection. J Am Acad Dermatol. 2020 [Epub ahead of print], doi: 10.1016/j.jaad.2020.07.013, indexed in Pubmed: 32652193.

26. Longo C, Specchio F, Ribero S, et al. Dermoscopy of small-size basal cell carcinoma: a case-control study. J Eur Acad Dermatol Venereol. 2017; 31(6): e273-e274, doi: 10.1111/jdv.13988, indexed in Pubmed: 27685248.

27. Takahashi A, Hara H, Aikawa M, et al. Dermoscopic features of small size pigmented basal cell carcinomas. J Dermatol. 2016; 43(5): 543-546, doi: 10.1111/1346-8138.13173, indexed in Pubmed: 26458728.

28. Liopyris K, Navarrete-Dechent $\mathrm{C}$, Yélamos $\mathrm{O}$, et al. Clinical, dermoscopic and reflectance confocal microscopy characterization of facial basal cell carcinomas presenting as small white lesions on sun-damaged skin. Br J Dermatol. 2019; 180(1): 229-230, doi: 10.1111/bjd.17241, indexed in Pubmed: 30239981.

29. di Meo N, Damiani G, Vichi S, et al. Interobserver agreement on dermoscopic features of small basal cell carcinoma ( $<5 \mathrm{~mm}$ ) among low-experience dermoscopists. J Dermatol. 2016; 43(10): 1214-1216, doi: 10.1111/1346-8138.13426, indexed in Pubmed: 27129742.

30. Carducci M, Bozzetti M, de Marco G, et al. Preoperative margin detection by digital dermoscopy in the traditional surgical excision of cutaneous squamous cell carcinomas. J Dermatolog Treat. 2013; 24(3): 221-226, doi: 10.3109/09546634.2012.672711, indexed in Pubmed: 22390630.

31. Carducci M, Bozzetti M, De Marco G, et al. Usefulness of margin detection by digital dermoscopy in the traditional surgical excision of basal cell carcinomas of the head and neck including infiltrative/morpheaform type. J Dermatol. 2012; 39(4): 326-330, doi: 10.1111/j.1346-8138.2011.01449.x, indexed in Pubmed: 22150641.

32. Conforti C, Giuffrida R, Zalaudek I, et al. Dermoscopic Findings in the Presurgical Evaluation of Basal Cell Carcinoma. A Prospective Study. Dermatol Surg. 2021;47(2): e37-e41, doi: 10.1097/DSS.0000000000002471, indexed in Pubmed: 32804889.

33. Hamilko de Barros M, Conforti C, Giuffrida R, et al. Clinical usefulness of dermoscopy in the management of lentigo maligna melanoma treated with topical imiquimod: A case report. Dermatol Ther. 2019; 32(5): e13048, doi: 10.1111/dth.13048, indexed in Pubmed: 31365164.

34. Blum A, Hofmann-Wellenhof R, Marghoob AA, et al. Recurrent melanocytic nevi and melanomas in dermoscopy: results of a multicenter study of the International Dermoscopy Society. JAMA Dermatol. 2014; 150(2): 138-145, doi: 10.1001/jamadermatol.2013.6908, indexed in Pubmed: 24226788.

35. Avilés-Izquierdo JA, Ciudad-Blanco C, Sánchez-Herrero A, et al. Dermoscopy of cutaneous melanoma metastases: A color-based pattern 
classification. J Dermatol. 2019; 46(7): 564-569, doi: 10.1111/13468138.14926, indexed in Pubmed: 31120139.

36. Farnetani F, Manfredini M, Longhitano S, et al. Morphological classification of melanoma metastasis with reflectance confocal microscopy. J Eur Acad Dermatol Venereol. 2019; 33(4): 676-685, doi: 10.1111/ jdv.15329, indexed in Pubmed: 30394598.

37. Moscarella E, Rabinovitz H, Puig S, et al. Multiple primary melanomas: do they look the same? Br J Dermatol. 2013; 168(6): 1267-1272, doi: 10.1111/bjd.12260, indexed in Pubmed: 23374221.

38. De Giorgi V, Salvini C, Sestini $\mathrm{S}$, et al. Triple synchronous cutaneous melanoma: a clinical, dermoscopic, and genetic case study. Dermatol Surg. 2007; 33(4): 488-491, doi: 10.1111/j.1524-4725.2007.33098.x, indexed in Pubmed: 17430385.

39. Rutkowski P, Owczarek W, Nejc D, et al. Skin carcinomas. Oncol Clin Pract. 2018; 14(3): 129-147, doi: 10.5603/OCP.2018.0019.

40. Husein-ElAhmed H, Fernandez-Pugnaire MA. Dermatoscopy-guided therapy of pigmented basal cell carcinoma with imiquimod. An Bras Dermatol. 2016; 91(6): 764-769, doi: 10.1590/abd1806-4841.20165255, indexed in Pubmed: 28099598.

41. Diluvio L, Bavetta M, Di Prete M, et al. Dermoscopic monitoring of efficacy of ingenol mebutate in the treatment of pigmented and non-pigmented basal cell carcinomas. Dermatol Ther. 2017; 30(1), doi: 10.1111/dth.12438, indexed in Pubmed: 27860083.

42. Mainetti C, Guillod C, Leoni-Parvex S. Successful Treatment of Relapsing Bowen's Disease with Ingenol Mebutate: The Use of Dermoscopy to Monitor the Therapeutic Response. Dermatology. 2016; 232 Suppl 1: 9-13, doi: 10.1159/000447389, indexed in Pubmed: 27513936.

43. Tognetti $L$, Cinotti E, Fiorani $D$, et al. Long-term therapy of multiple basal cell carcinomas: Clinicodermoscopic score for monitoring of intermittent vismodegib treatment. Dermatol Ther. 2019; 32(6): e13097, doi: 10.1111/dth.13097, indexed in Pubmed: 31612619.

44. Navarrete-Dechent C, Cordova M, Liopyris K, et al. In vivo imaging characterization of basal cell carcinoma cutaneous response to high dose ionizing radiation therapy: A prospective study of reflectance confocal microscopy, dermoscopy, and ultrasound. J Am Acad Dermatol. 2020 [Epub ahead of print], doi: 10.1016/j.jaad.2020.07.130, indexed in Pubmed: 32827607.

45. Richtig E, Arzberger E, Hofmann-Wellenhof R, et al. Assessment of changes in lentigo maligna during radiotherapy by in-vivo reflectance confocal microscopy: a pilot study. Br J Dermatol. 2015; 172(1): 81-87, doi: 10.1111/bjd.13141, indexed in Pubmed: 24889911.

46. Ballester Sánchez R, Pons Llanas O, Pérez Calatayud J, et al. Dermoscopy margin delineation in radiotherapy planning for superficial or nodular basal cell carcinoma. Br J Dermatol. 2015; 172(4): 1162-1163, doi: 10.1111/bjd.13402, indexed in Pubmed: 25204461.

47. Rajczykowski M, Kaminska-Winciorek G, Nowara E, et al. Dermoscopic assessment of skin toxicities in patients with melanoma during treatment with vemurafenib. Postepy Dermatol Alergol. 2018; 35(1): 39-46, doi: 10.5114/ada.2018.73163, indexed in Pubmed: 29599670.

48. Cohen PR, Bedikian AY, Kim KB. Appearance of New Vemurafenib-associated Melanocytic Nevi on Normal-appearing Skin: Case Series and a Review of Changing or New Pigmented Lesions in Patients with Metastatic Malignant Melanoma After Initiating Treatment with Vemurafenib. J Clin Aesthet Dermatol. 2013; 6(5): 27-37, indexed in Pubmed: 23710269.

49. https://dermoscopedia.org/. 